\title{
Aproximación al análisis cualitativo de redes sociales. Experiencias en el estudio de redes personales mediante Ego.Net.QF
}

\author{
Kerstin Hein-Instituto de Juventud Alemán, Alemania ${ }^{1}$ \\ Ana Cárdenas Tomažič-Universidad Diego Portales, Chile \\ Karla Henríquez- Universidad de Santiago de Chile, Chile \\ Santiago Valenzuela- Universidad de Santiago de Chile, Chile
}

\begin{abstract}
Resumen
El texto realiza una aproximación a la práctica de la investigación sobre redes sociales desde una perspectiva cualitativa. Dentro de este contexto destaca el uso del modelo de círculos concéntricos o tarjetas de red como principal herramienta para la visualización de las redes sociales de una persona. El presente artículo gira en torno a la aplicación e interpretación de un modelo particular de círculos concéntricos denominado Ego.Net.QF. El propósito de la discusión es analizar y reflexionar sobre los potenciales y las limitaciones de este instrumento a partir de distintas experiencias prácticas de aplicación.
\end{abstract}

Palabras clave: Redes personales - metodología cualitativa - tarjetas de red Ego.Net.QF.

\begin{abstract}
The contribution seeks to make an approach to the praxis of qualitative network research. Within this context, the article focuses on the application of network cards. Network cards are based on the model of concentric circles and represent the main tool to visualize the social networks of a single person. The present article pays special attention to the application and interpretation of a particular model of concentric circles called Ego.Net.QF. The purpose of the discussion is to analyze and reflect on the potentials and limitations of this instrument considering different experiences of practical application.
\end{abstract}

Key words: Personal networks - Qualitative methodology - Network cards Ego.Net.QF 


\section{I ntroducción}

Distintos autores asocian el inicio del análisis de redes sociales con los trabajos de un grupo de académicos de la Universidad de Harvard durante los años 1970. A partir de entonces se ha concebido el análisis de redes sociales como el estudio de las características formales y estructurales de conglomerados sociales mediante el uso de procedimientos algebraicos (Diaz-Bone, 2007; Franke \& Wald, 2006; Hollstein, 2006, 2010; Jansen, 2003; Raab, 2010). No obstante, durante las últimas décadas se puede observar una progresiva demanda por complementar el análisis cuantitativo de redes sociales con procedimientos cualitativos de investigación social con el fin de incluir de mejor manera la perspectiva subjetiva y agencia de los actores involucrados (Diaz-Bone, 2007; Franke \& Wald, 2006; Hollstein, 2006; Jansen, 2003). El resultado ha sido una pluralidad teórica, metodológica y técnica en el abordaje de las redes sociales (Gualda Caballero, 2005).

El estudio de redes sociales mediante métodos cualitativos constituye aún un ámbito poco definido. De hecho, Diaz-Bone (2007) cuestiona la existencia de un análisis de redes propiamente cualitativo, ya que existen pocos estudios de relaciones sociales que sean realmente cualitativos de principio a fin. En este sentido, lo cualitativo hace más bien referencia a la utilización de técnicas específicas que funcionan como suplemento del análisis cuantitativo de redes (DiazBone, 2007; Franke \& Wald, 2006; Hollstein, 2006).

De modo similar, Franke y Wald (2006) puntualizan que casi no existen procedimientos de análisis cualitativo que sean específicos para el estudio de redes sociales. Lo que se observa más bien es la aplicación de técnicas cualitativas clásicas y genéricas tanto en la recolección como en el análisis de los datos (DiazBone, 2007; Franke \& Wald, 2006; Olivier, 2013). Por otro lado, existe un uso frecuente de instrumentos estandarizados, los cuales son adaptados para la recogida de datos en el marco de un estudio cualitativo. Debido a esta situación no siempre cumplen con los criterios de apertura exigidos por la investigación cualitativa (Diaz-Bone, 2007; Franke \& Wald, 2006).

En el presente artículo revisaremos de qué manera ha sido concebida hasta el momento la investigación cualitativa de redes sociales. Posteriormente nos referiremos a algunas experiencias prácticas en la recogida y análisis de relaciones

1 Contacto: Kerstin Hein. Instituto de Juventud Alemán, Nockherstr. 2, 81541 Munich, Alemania Direcciones electrónicas: khein@dji.de, kerstinhein@gmail.com 
personales mediante tarjetas de red con el fin de discutir la finalidad, los potenciales y limitaciones de tal instrumento para el estudio cualitativo de redes sociales.

\section{I nvestigación cualitativa de redes sociales como laboratorio de experimentación}

De acuerdo con Straus (2010), el estado actual de la investigación cualitativa de redes sociales aún constituye un laboratorio de experimentación. No sólo no existe claridad con respecto a lo que constituye una investigación cualitativa de redes sociales. También se observa un alto grado de improvisación en las técnicas de recolección y análisis utilizados. De este modo, cuando se habla de estudios cualitativos de redes sociales, por lo general se hace referencia a estudios de metodología mixta (Diaz-Bone, 2007; Franke \& Wald, 2006; Gualda Caballero, 2005; Hollstein, 2010).

En diseños de investigación mixtos las técnicas cualitativas y cuantitativas se complementan mutuamente. Lo típico es recoger la información mediante cuestionarios e instrumentos que permitan reconstruir y visualizar las redes sociales de una persona. Luego se complementa tal información mediante una entrevista semi-estructurada o abierta que permite obtener información acerca de la perspectiva subjetiva y las lógicas de acción de los entrevistados (Aguilar, 2005; Diaz-Bone, 2007; Franke \& Wald, 2006; Hollstein, 2006, 2010; Olivier, 2013; Schütz, 2006; Straus, 2006, 2013).

De acuerdo con esta perspectiva, las técnicas cualitativas tienen la capacidad de enriquecer y complementar los datos cuantitativos acerca de las redes sociales. A pesar de la pluralidad teórica y metodológica de los diseños mixtos, la literatura tiende a clasificar este tipo de estudios como cualitativos, ya que recogen datos de manera abierta y reconstruyen el significado de las relaciones sociales mediante el uso de técnicas de interpretación (Diaz-Bone, 2007; Franke \& Wald, 2006; Hollstein, 2006; Olivier, 2013).

Hollstein (2010) distingue tres distintas formas de combinar las perspectivas metodológicas cualitativas y cuantitativas al interior de una investigación sobre redes sociales. En este sentido, es posible hablar en primer lugar de un diseño mixto secuencial y explicativo, el cual comienza con una recogida cuantitativa de los datos. La información obtenida de esta manera es posteriormente complementada y profundizada mediante entrevistas cualitativas. Este tipo de procedimiento puede ser observado en McCarty, Molina, Aguilar y Rota (2007) y McCarty (2002). En ambos casos se utilizan procedimientos cuantitativos con el fin de construir una representación gráfica de las redes sociales de una persona. La 
visualización de las redes personales es utilizada a su vez para preparar una entrevista cualitativa, en la cual el investigador obtiene información relevante para la interpretación y validación de los datos relacionales.

Otra forma de articular las distintas perspectivas de investigación consiste en implementar un diseño mixto secuencial exploratorio. Tales estudios comienzan con una exploración de tipo cualitativa, por ejemplo a través de una observación participante, a la cual sigue la utilización de instrumentos cuantitativos como lo puede ser la aplicación de una encuesta. Un ejemplo para la articulación secuencial exploratoria entre lo cualitativo y lo cuantitativo es el procedimiento propuesto por Mckether, Gluesing y Riopelle (2009), el cual consiste en extraer, codificar y visualizar datos relacionales contenidos en entrevistas narrativas mediante el uso de distintos programas de software.

Finalmente, se puede distinguir un tipo de diseño mixto paralelo, en el cual se articulan de manera paralela y compleja elementos cualitativos y cuantitativos de investigación. Este tipo de diseño requiere una triangulación sistemática de los datos con el fin de garantizar un efecto de sinergía (Flick, 2000, 2004; Franke \& Wald, 2006; Hollstein, 2010).

Más allá de la distinción entre una perspectiva cualitativa y cuantitativa, investigadores han indicado que una característica del estudio de redes sociales es su pluralidad metodológica y técnica (Gualda Caballero, 2005; Hollstein, 2010). Otros autores como Schönhuth y Gamper (2013) han afirmado que las distinciones entre lo cualitativo y cuantitativo se diluyen a favor de lograr una adecuada visualización de las redes. La visualización de las redes sociales puede constituir el resultado de una investigación, ser utilizada como estímulo en el contexto de una entrevista (Aguilar, 2005; Noack \& Schmidt, 2013; Straus, 2010) o ser objeto de reflexión por parte de personas o grupos (Corsten, 2007; Scheibelhofer, 2006; Schiffer \& Hauck, 2010; Schönhuth, 2013).

No obstante lo anterior, durante los últimos años ha aumentado la demanda por continuar desarrollando la investigación cualitativa de redes sociales más allá de su rol complementario con respecto al análisis cuantitativo (Olivier, 2013; Straus, 2013). Esto ha implicado por sobre todo el interés por adaptar o desarrollar instrumentos específicos para la recolección de datos desde una perspectiva metodológica cualitativa. En menor medida también ha significado el desarrollo de procedimientos cualitativos para el análisis y la interpretación de los datos.

A continuación centraremos nuestra atención en la reconstrucción de redes sociales en el marco de la investigación cualitativa mediante el uso de tarjetas de red. Discutiremos la aplicación de tal instrumento a la luz de nuestra experiencia en 
diferentes proyectos de investigación cualitativa llevados a cabo en el contexto chileno.

\section{El uso de tarjetas de red para el estudio de redes egocéntricas}

El uso de tarjetas de red para la visualización de redes egocéntricas ha adquirido especial importancia en el contexto de la investigación cualitativa en países de habla alemana (Diaz-Bone, 2007; Franke \& Wald, 2006; Schönhuth, 2013; Schönhuth \& Gamper, 2013; Straus, 2010, 2013). Las tarjetas de red constituyen una forma de visualización que ha sido utilizada tanto en la investigación cuantitativa como cualitativa con el fin de reconstruir las relaciones sociales en torno a un actor o ego. En este sentido, también se habla de la reconstrucción de redes egocéntricas, en cuyo centro puede figurar una persona, un grupo o una organización/institución. El contraste lo constituye la representación de una red total, la cual es generada de manera descentralizada considerando la perspectiva de varios o todos los actores involucrados (Diaz-Bone, 1997; Jansen, 2003; Schönhuth, 2013; Straus, 1994, 2002, 2010, 2013; Wolf, 2010).

La mayoría de las tarjetas de red consiste en un diagrama de círculos concéntricos, cuyo punto central representa a ego. El investigador solicita a ego que seleccione ciertas personas, grupos u organizaciones/instituciones (alteri) de acuerdo con un concepto generador de nombres. El concepto generador de nombres es determinado de antemano por el investigador en concordancia con sus objetivos de investigación. A continuación se inscriben las personas, grupos $u$ organizaciones/instituciones seleccionados a menor o mayor distancia del centro según su cercanía o lejanía con respecto a ego (Diaz-Bone, 1997; Straus, 2002, 2010; Wolf, 2010).

La idea básica de las tarjetas de red también ha sido adaptada de manera de poder dar cuenta de la distribución geográfica de los nodos de la red. Es así como Olivier (2013) ha asignado a los círculos concéntricos atributos de cercanía o distancia geográfica con el fin de conocer, si las personas que conoce ego se encuentran en el mismo vecindario, en la misma localidad o ciudad, en otra ciudad del país o fuera del país, mientras que Kesselring (2006) explora el potencial de movilidad de sus entrevistados por medio de la distribución de lugares en vez de personas a lo largo de los círculos concéntricos.

El grado de estructuración de las tarjetas varía de acuerdo con los objetivos de cada estudio, pudiendo identificarse diagramas estandarizados o semiestandarizados (Schönhuth \& Gamper, 2013). Un ejemplo de diagrama 
estandarizado es proporcionado por Kahn y Antonucci (1980), quienes atribuyen a cada círculo concéntrico una determinada categoría de atributos (muy cercano, cercano, poco cercano pero importante).

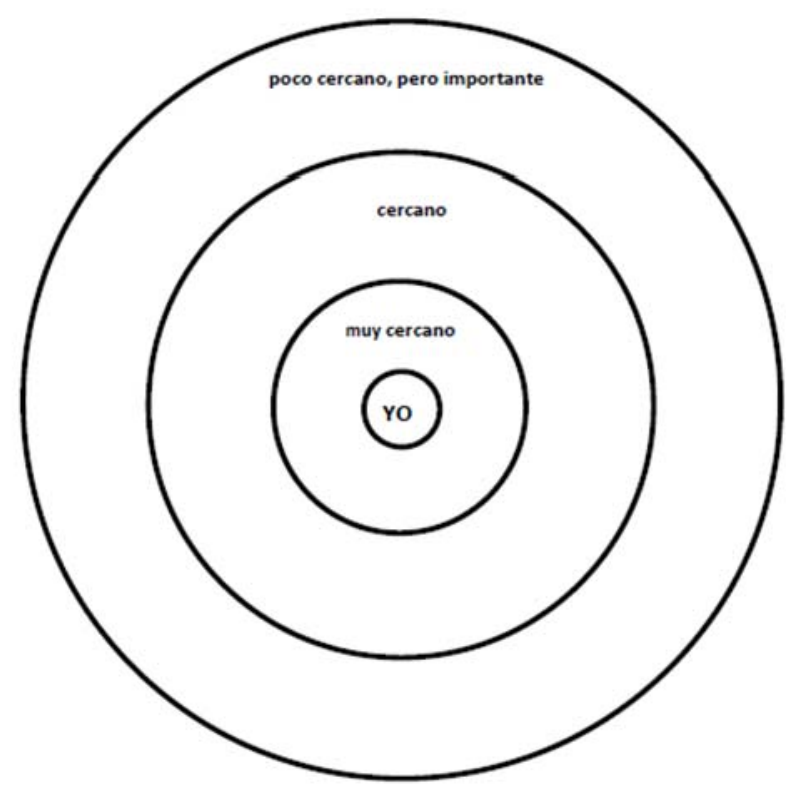

Gráfico 1. Modelo de círculos concéntricos según Kahn y Antonucci (1980).

Un diagrama semi-estandarizado también presenta cierto grado de estructuración como el uso de círculos concéntricos o segmentación del diagrama. Sin embargo, es utilizado de manera flexible y por lo general no determina categorías de atributos para los distintos círculos (Schönhuth \& Gamper, 2013).

De acuerdo con el principio de validez ecológica (Lamnek, 1995), investigadores cualitativos prefieren aplicar tarjetas de red semi-estandarizadas, puesto que éstas permiten mayor libertad de expresión y participación por parte del entrevistado. Por otra parte, la reconstrucción de las redes egocéntricas ocurre en el contexto de entrevistas cualitativas con el fin de poder contextualizar y complementar la visualización de las redes sociales con elementos narrativos (Diaz-Bone, 2007; Olivier, 2013). 


\section{Ego.Net.QF}

En nuestro caso hemos aplicado un tipo particular de tarjeta de red para la reconstrucción de redes egocéntricas denominado Ego.Net.QF. Ego.Net.QF ha sido desarrollado sobre la base del modelo de círculos concéntricos y adaptado especialmente para su uso en entrevistas cualitativas por un grupo de psicólogos comunitarios en Alemania (Straus, 1994, 2002, 2013).

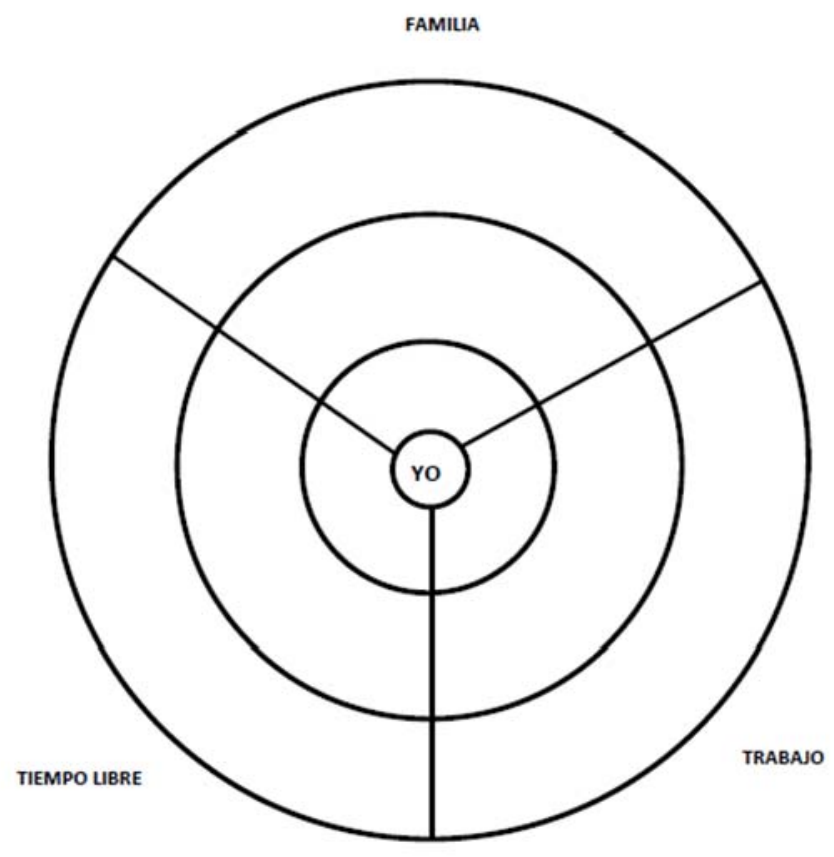

Gráfico 2. Ego.Net.QF según Straus (1994, 2002)

El instrumento fue concebido como una forma de visualización global de las redes sociales de un individuo y testeado en el marco de un estudio longitudinal sobre el desarrollo de la identidad en la modernidad tardía (cf. Keupp et al., 1999).

El objetivo inicial fue lograr desarrollar un instrumento para la visualización de redes sociales que fuese fácil de comprender y aplicar, y que por sobre todo no interrumpiese el flujo de la conversación durante la entrevista. Este último aspecto fue motivo de extensas discusiones, ya que investigadores cualitativos veían el

\footnotetext{
2 Ego.Net.QF constituye una técnica de recolección de datos diseñada para la reconstrucción y visualización de redes sociales en el contexto de la investigación cualitativa. Como tal debe ser distinguida del Software EgoNet utilizado para la recolección y el análisis de redes personales mediante el uso de cuestionarios y la aplicación de modelos matemáticos (http://sourceforge. net/projects/egonet/)
} 
análisis de redes como un elemento exógeno y se sentían inseguros con respecto al momento adecuado para su introducción en el marco de una entrevista. Por un lado se temía que el entrevistado enumerara las personas que conoce en vez de narrar algo sobre ellas. Por otro lado existía escepticismo con respecto al valor agregado de una visualización de las redes en comparación con una simple conversación sobre las relaciones sociales del entrevistado. En la medida que el instrumento fue más conocido y difundido, se pudo apreciar que no era motivo de interrupción de la entrevista, sino que al contrario funcionaba en sí mismo como estímulo narrativo (Straus, 1994, 2013).

La aplicación de Ego. Net.QF durante la entrevista resulta interactiva y lúdica, ya que el entrevistado utiliza elementos como alfileres, lápices de colores, hilos y etiquetas para reconstruir su red social personal. La utilización de este tipo de elementos garantiza la participación activa por parte del entrevistado y la flexibilidad del instrumento. La persona puede modificar la representación de su red social hasta el término de la entrevista. Durante la reconstrucción de la red el entrevistado es alentado a contar historias acerca de las personas identificadas y el tipo de relación que se mantiene con éstas (Straus, 1994, 2002).

A diferencia de otros métodos similares, Ego.Net.QF realiza una segmentación del diagrama de círculos concéntricos en sectores de la vida cotidiana como por ejemplo la familia, el tiempo libre o el trabajo. Esta segmentación se propuso, por un lado, con el fin de apoyar la enumeración de personas importantes de la red. Por otro lado, la segmentación también facilita la comparación entre distintas redes sociales en el marco de una investigación (Diaz-Bone, 2007; Straus, 1994, 2002). La segmentación del diagrama constituye el primer paso en la aplicación del instrumento. Se solicita al sujeto que divida el gráfico de acuerdo a los sectores relevantes de su vida cotidiana como si estos fuesen trozos de una torta. El tamaño del "pedazo de torta" dependerá de la importancia asignada al segmento (Straus, 1994, 2002).

Tras la segmentación del diagrama se pide al entrevistado nombrar todas aquellas personas que considera relevantes al interior de cada segmento. Aquí resulta importante recordar al entrevistado que la relevancia de una persona no necesariamente significa simpatía, ya que alguien puede ser importante, a pesar de ser una persona poco agradable. A continuación se solicita al entrevistado utilizar los alfileres para ubicar a sus alteri a mayor cercanía o lejanía con respecto a ego de acuerdo a la importancia atribuida. Al mismo tiempo se registra el nombre de las personas mediante etiquetas o un lápiz que sea fácil de borrar (Straus, 1994, 2002). 
Una vez reconstruida la red social es posible realizar preguntas concretas acerca de los nodos y las relaciones representadas. Este tipo de preguntas acerca de la función o de los atributos de los alteri también es utilizada en el marco de investigaciones cuantitativas. En este caso las preguntas son formuladas de manera estandarizada (Wolf, 2010), mientras que en el marco de la investigación cualitativa se espera que las preguntas funcionen como estímulos narrativos (Straus, 1994, 2002).

\section{Experiencias en la aplicación de Ego.Net.QF}

Hemos tenido la oportunidad de utilzar Ego.Net.QF en distintas investigaciones cualitativas en el contexto chileno. Una de estas experiencias corresponde a la utilización del instrumento en el marco de un estudio sobre jóvenes inmigrantes de origen latinoamericano en Chile (cf. Hein, 2012, 2013)3. El objetivo principal de la investigación fue lograr describir y comprender la experiencia de estos jóvenes en el sistema educacional chileno y la forma en que significan y manejan la transición de la escuela al trabajo. Dentro de este marco utilizamos la tarjeta de red con el fin de identificar los recursos sociales, materiales y culturales con los cuales contaban los jóvenes inmigrantes en el contexto chileno.

Los datos fueron recogidos principalmente mediante entrevistas biográficas narrativas. La reconstrucción de las redes sociales fue llevada a cabo mediante la utilización de Ego.Net.QF y considerada como complemento de las entrevistas biográficas. En vista de la experiencia en estudios anteriores (Hein, 2006), decidimos aplicar las tarjetas de red en un encuentro posterior y separado de las entrevistas con el fin de otorgarle mayor espacio a la conversación sobre las redes sociales. Al descomprimir la entrevista biográfica y realizar la visualización de las relaciones sociales en un encuentro separado también logramos evitar el agotamiento de los participantes.

La aplicación de Ego. Net.QF fue llevada a cabo de acuerdo a lo propuesto por Straus $(1994 ; 2002)$ con la diferencia de que utilizamos un diagrama de menor tamaño y prescindimos de la utilización de hilos para la demarcación de los segmentos. Estas modificaciones fueron introducidas, por un lado, con el fin de facilitar el transporte de los materiales a los lugares de entrevista. En cuanto a la

\footnotetext{
${ }^{3}$ El estudio corresponde al proyecto FONDECYT No. 11085001 “Migración y Transición: Estudio biográfico interpretativo acerca de la significación y manejo de la Transición de la escuela al trabajo por parte de jóvenes con trasfondo migratorio de origen latinoamericano en Chile" y fue financiado por la Comisión Nacional de Investigación Científica y Tecnológica de Chile.
} 
segmentación del diagrama optamos por reemplazar los hilos por palillos con el objetivo de simplificar la aplicación del instrumento. También decidimos predefinir algunos segmentos (familia, educación, trabajo) para facilitar la comparación de las redes sociales de los distintos casos. No obstante, los participantes podían agregar o quitar segmentos, si lo consideraban pertinente. Los nodos de la red fueron representados inicialmente por pinchos. Palillos y pinchos permitieron una flexible modificación de la red hasta el final de la entrevista. Recién entonces se utilizaron lápices para fijar los segmentos y las personas. Tras la finalización del diagrama se realizaron preguntas sobre el apoyo social y los recursos materiales y culturales vinculados a la red personal del entrevistado.

Una particularidad del presente estudio fue que la recolección de datos se llevó a cabo en dos etapas. La primera recogida de datos fue realizada mientras los jóvenes inmigrantes se encontraban en el último año de educación secundaria, mientras que la segunda etapa tuvo lugar un año después que hubiesen finalizado la escuela. Este diseño permitió observar no solo las diferencias entre las redes sociales de los distintos participantes sino también los cambios ocurridos en la conformación de las redes de cada caso en el transcurso del tiempo.

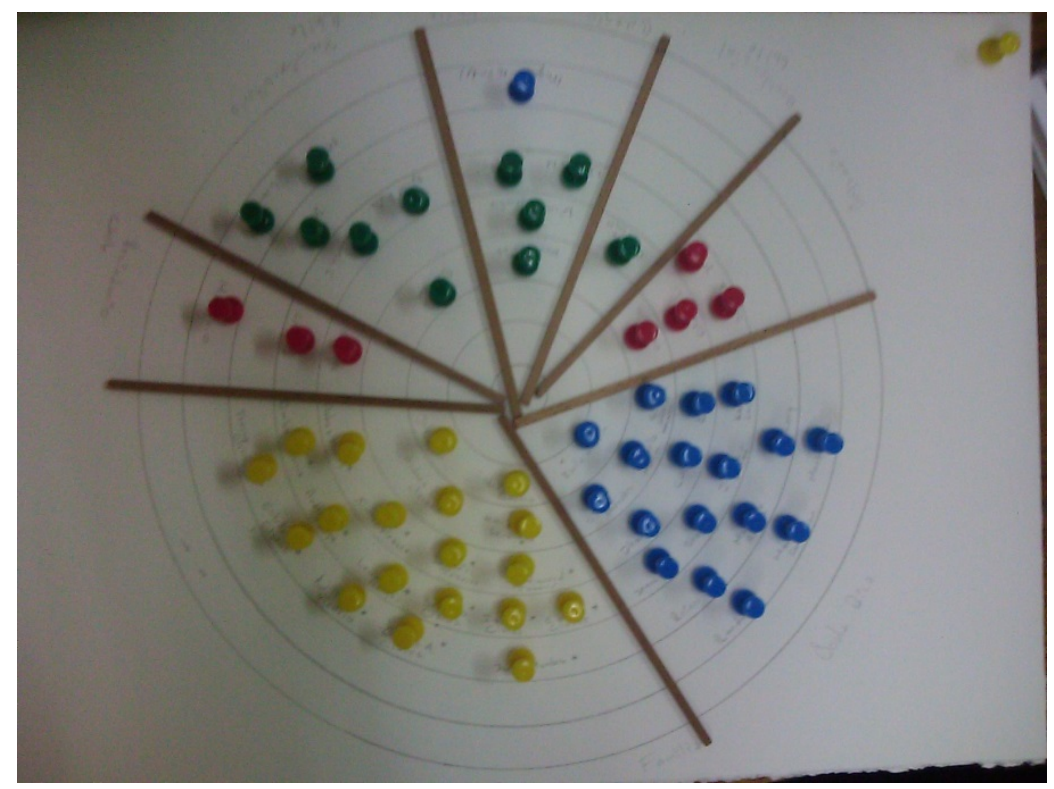

Diagrama 1. Aplicación de Ego.Net.QF con palillos y pinchos

Otra investigación en la cual hemos tenido la oportunidad de volver a aplicar las tarjetas de red corresponde a un estudio sobre los modos en que madres de diferentes niveles educacionales con hijos/as en la primera infancia concilian 
responsabilidades familiares y laborales (Cárdenas, 2012)4. Al igual que en el caso anterior, la reconstrucción de la red egocéntrica fue entendida como una técnica complementaria a la entrevista biográfica. Uno de los objetivos específicos asociados a la reconstrucción de las redes sociales de las entrevistadas fue comprender el modo en que ellas logran conciliar trabajo y familia. Otro objetivo de la aplicación de las tarjetas de red fue lograr identificar a las personas e instituciones que apoyan a las madres en la organización de su día a día. Por lo general, la reconstrucción de las redes sociales fue realizada a continuación de la entrevista biográfica. Sin embargo, también hubo ocasiones en que el instrumento fue utilizado como estímulo narrativo durante el desarrollo de la entrevista.

En cuanto a la segmentación del diagrama, se solicitó a las entrevistadas identificar y reconstruir sus redes sociales en las esferas del trabajo y la familia con la posibilidad o no de establecer un límite entre ambos segmentos. El límite era establecido de manera provisoria mediante fósforos durante la entrevista. Hacia el final del encuentro, las participantes podían trazar o no el límite definitivo mediante el uso de un lápiz. Este procedimiento permitió observar, si los ámbitos de la familia y el trabajo se encontraban claramente delimitados o no. Asimismo fue posible registrar el modo en que las participantes jerarquizaban ambas esferas. Al respecto cabe destacar, que varias entrevistadas decidieron no trazar un límite entre familia y trabajo. Este resultado puede interpretarse como una forma de comprender y organizar ambas esferas de manera conjunta en un contexto de creciente participación laboral femenina, pero de escasa redefinición de los roles tradicionales de género al interior de la familia (Programa de las Naciones Unidas para el Desarrollo (PNUD), 2010; Programa de las Naciones Unidas para el Desarrollo (PNUD) \& Organización Internacional del Trabajo (OIT), 2009; Todaro \& Yañez, 2004).

\section{Reflexiones sobre la aplicación de Ego.Net.QF}

Una característica común en el uso de las tarjetas de red a lo largo de las investigaciones mencionadas fue que la reconstrucción de redes sociales siempre se concibió como un método complementario con respecto a las entrevistas cualitativas. Dicho de otra manera, las relaciones sociales no constituyeron un

\footnotetext{
${ }^{4}$ El estudio corresponde al proyecto FONDECYT No. 11110420 “Trabajo y Maternidad: Estudio biográfico interpretativo sobre la articulación del ámbito laboral y familiar en mujeres con hijos en la primera infancia" y fue financiado por la Comisión Nacional de Investigación Científica y Tecnológica de Chile.
} 
objeto de investigación en sí. No obstante, la reconstrucción de las relaciones personales resultó ser un valioso material de apoyo, tanto para la interpretación de las entrevistas como también para la comprensión de cada caso.

Gracias a la aplicación de las tarjetas de red fue posible conocer de manera concreta las relaciones sociales en las cuales se insertaban los entrevistados, lo que a su vez permitió traducir afirmaciones realizadas de manera abstracta durante la entrevista a un nivel cotidiano y concreto. Por ejemplo, varios jóvenes inmigrantes afirmaron durante la entrevista que se sentían discriminados en Chile, sin poder especificar el origen concreto de su malestar. No obstante, al reconstruir las redes sociales, los participantes fueron capaces de identificar personas y situaciones particulares en las cuales se sentían discriminados.

También en el caso del estudio sobre la conciliación entre responsabilidades familiares y laborales, la reconstrucción de las redes sociales permitió identificar personas específicas y su relevancia como elemento de apoyo para las madres. Algunas de estas personas no eran mencionadas en el contexto de la entrevista, sino que aparecían recién en la reconstrucción de las redes sociales. Por ejemplo, una entrevistada indicó durante la reconstrucción de sus redes a una secretaria quien en ocasiones llevaba a los niños a la casa mientras la madre aún se encontraba en reunión. Como este tipo de apoyo era más bien ocasional, la participante había olvidado mencionarla en el marco de la entrevista, mientras que salió a la luz durante la aplicación de la tarjeta de red.

En suma, la tarjeta de red fue más que una mera técnica de recolección de datos. Su aplicación demostró que la reconstrucción de las redes sociales también constituye un importante instrumento de aprendizaje y reflexión para los entrevistados. Este aspecto fue especialmente destacado por parte de las participantes en el estudio sobre la conciliación de responsabilidades laborales y familiares. De acuerdo a estas madres, la visualización de las redes sociales les permitió desarrollar una mirada más reflexiva con respecto al modo en que organizan su vida cotidiana y los cambios ocurridos desde el nacimiento de sus hijos/as.

Por otro lado, en el caso de los jóvenes inmigrantes en Chile, la aplicación de las tarjetas de red demostró ser particularmente útil para la identificación de los recursos materiales, sociales y culturales de los entrevistados. De este modo fue posible darse cuenta que las relaciones familiares y de amistad con otros inmigrantes resultaban ser especialmente relevantes para el bienestar subjetivo de los entrevistados, mientras que las relaciones discriminatorias ocurrían de preferencia entre pares del contexto escolar (Hein, 2013). 
También resulta importante destacar el rol que cumplieron las tarjetas de red como estímulo narrativo en el marco de las entrevistas. En este sentido, el reconstruir y visualizar las relaciones sociales permitió estimular los recuerdos y narraciones de los participantes con respecto a sus experiencias pasadas y cotidianas. Este tipo de relatos no siempre aparecieron en el contexto de las entrevistas biográficas, lo que pensamos puede estar vinculado con la expectativa sociocultural de tener que construir un relato coherente (Rosenthal, 2005).

Por otra parte, cabe señalar que la utilización de Ego. Net.QF no sólo tuvo aspectos positivos, sino que también presentó algunas limitaciones. Entre las limitaciones observadas destacan ciertas dificultades de comprensión tanto en relación al sentido del instrumento como también con respecto al procedimiento de aplicación.

En el marco del estudio sobre conciliación de familia y trabajo pudimos observar que las participantes con educación secundaria completa y sin estudios posteriores mostraron dificultades en comprender las instrucciones. En estos casos, se complementaron y especificaron las instrucciones mediante el uso de ejemplos concretos. Una vez que las participantes lograron comprender el sentido y el potencial de autorreflexión a partir de la visualización de sus redes sociales, se mostraron altamente motivadas en el uso del instrumento.

Otra traba relativamente frecuente a la cual nos vimos enfrentados en la aplicación de Ego. Net. QF tuvo relación con la segmentación del diagrama. Especialmente en la investigación sobre migrantes latinoamericanos en Chile observamos cierto desconcierto ante la división de la red. La segmentación de la vida cotidiana de acuerdo a ítemes preestablecidos como familia, escuela o tiempo libre parecía no corresponder con la forma en que los entrevistados comprendían su diario vivir. En este sentido observamos que varios entrevistados se resistieron a la segmentación predefinida y optaron por establecer divisiones propias. Como consecuencia obtuvimos redes con sectores muy diferentes y poco comparables entre sí. Es así que en el caso de los jóvenes inmigrantes llegamos a la conclusión que la segmentación del diagrama más bien perturbó la construcción de las redes sociales y no representó un aporte significativo para la interpretación de los datos.

Por otra parte, encontramos que las tarjetas de red establecen varias restricciones en la forma de representación de las relaciones sociales, lo cual favorece un análisis cuantitativo, pero limita la interpretación del material desde una perspectiva cualitativa. En este sentido tuvimos la impresión de que el grado de estructuración de las tarjetas de red restringió la libertad de expresión subjetiva de los entrevistados, lo que a su vez limitó la interpretación de estos datos. Es posible que esta sea una de las razones por las cuales los estudios cualitativos hayan tendido a 
centrarse en la interpretación de los relatos acerca de las redes más que en el análisis de su representación gráfica.

La visualización de las redes sociales mediante el diagrama de círculos concéntricos presentó especiales limitaciones cuando el número de alteri era muy extenso, lo cual fue bastante frecuente en el estudio sobre jóvenes inmigrantes. En este caso, la entrevista muchas veces derivó en la enumeración de personas sin poder profundizar en su relación con ego. Esto a su vez se traducía en un gráfico sobrecargado que hacía difícil una interpretación desde el punto de vista cualitativo. Esta limitación no fue observada en el caso de las trabajadoras madres, lo que posiblemente tiene relación con el hecho de que las redes sociales tienden a disminuir con la edad (Wolf, 2010).

Finalmente, desde el punto de vista de los aspectos éticos asociados al análisis de las tarjetas de red, cabe destacar la dificultad de asegurar el anonimato de los alteri en el marco de la visualización. Esto adquiere especial importancia cuando se reconstruyen las redes sociales mediante el uso de lápiz y papel. También es relevante en redes sociales muy amplias, cuando resulta difícil reemplazar el nombre de las personas por sus roles sociales, como por ejemplo hermano o tío. Esta situación restringe el uso de este tipo de gráficos en presentaciones o publicaciones.

\section{Entre narración y visualización: Acerca del análisis cualitativo de las redes sociales}

Tal como hemos podido apreciar anteriormente, el aumento del interés por el análisis cualitativo de las redes sociales ha gatillado una intensa discusión sobre las técnicas de recolección de datos y su adaptación para los fines de la investigación cualitativa. Lamentablemente no ha sucedido lo mismo con el análisis cualitativo de las redes sociales. Hasta el momento no existe un procedimiento de análisis cualitativo específico para este tipo de datos (Olivier, 2013).

La mayoría de los estudios cualitativos que han incluido un análisis de redes sociales han recurrido a métodos cuantitativos para el análisis de los datos. Por otra parte, aquellos estudios que efectivamente han realizado un abordaje cualitativo de las redes sociales han interpretado los datos mediante métodos clásicos y genéricos tales como el análisis de contenido o la teoría fundada. Cabe destacar que estas herramientas han sido aplicadas principalmente para analizar los relatos acerca de las redes, mientras que falta un procedimiento cualitativo para intepretar la 
visualización de las mismas (Noack \& Schmidt, 2013; Olivier, 2013; Schönhuth, 2013).

Straus (1994; 2002; 2013) confirma esta apreciación al plantear que la visualización de las redes sociales ha sido utilizada principalmente como complemento para la interpretación cualitativa del texto transcrito. En el fondo se ha utilizado la visualización como estímulo narrativo durante la entrevista y luego analizado de manera cualitativa el relato (Straus, 2010). No obstante, durante los últimos años ha aumentado la demanda por desarrollar un procedimiento que no solo tome en cuenta los relatos, sino que también permita analizar de manera cualitativa la representación gráfica de las redes (Straus, 2013).

Una forma de articular el análisis de la visualización con el análisis del texto ha consistido en utilizar las representaciones gráficas de las relaciones sociales como fundamento para generar hipótesis o conceptos sensibilizadores acerca de las redes. Estos conceptos sensibilizadores son utilizados posteriormente para guiar la interpretación de los relatos (Olivier, 2013). Por otro lado, Lozares y Verd (2008) proponen una interesante forma de reconstrucción de las redes sociales a partir de la entrevista biográfica, mientras que Straus (2010) plantea la idea de confrontar lo dicho durante la entrevista con la visualización de las redes. No obstante, a pesar de estas iniciativas, hasta el momento no ha sido posible resolver de manera convincente la tensión entre la visualización de las redes sociales y las narraciones sobre éstas (Olivier, 2013).

En nuestro caso tampoco hemos podido resolver de manera adecuada la tensión entre el texto transcrito y la visualización de las redes sociales. En todos los estudios mencionados hemos focalizado nuestra atención en la interpretación de lo dicho sobre las redes, más que en el análisis de su representación gráfica. Aún así, los datos obtenidos mediante la aplicación de las tarjetas de red fueron cualitativamente distintos a los datos obtenidos durante las entrevistas y permitieron reconstruir las relaciones sociales de los entrevistados de manera complementaria a la entrevista. Al agrupar lo dicho en categorías amplias como las relaciones con los padres o en el vecindario también fue posible comparar las redes sociales entre sí.

Finalmente, cabe señalar que el uso de redes egocéntricas en el marco de la investigación cualitativa frecuentemente descuida la reconstrucción de las relaciones entre los alteri. Dicho de otra manera, o bien no se recoge o se recoge de manera insuficiente la relación entre las personas que conoce ego. Esto restringe las posibilidades de interpretación de los datos en términos de la 
interrelación de los nodos (Diaz-Bone, 2007) y dificulta identificar la posición de ego al interior de sus redes sociales (Straus, 2010).

\section{Alternativas a las tarjetas de red: Herramientas digitales, dibujos de redes sociales y juego de redes socio-espaciales}

El uso de tarjetas de red no constituye la única alternativa para el estudio de redes sociales desde un punto de vista cualitativo. Especialmente durante los últimos años, se observa una importante difusión en el uso de software para la reconstrucción de las relaciones sociales de una persona, como por ejemplo VennMaker. En general, ha habido una positiva recepción de estas alternativas digitales en el contexto de la investigación cualitativa (Schönhuth, 2013; Straus, 2010).

La mayoría de las herramientas digitales retoma la idea de los círculos concéntricos para la reconstrucción de redes egocéntricas. No obstante, permite gran flexibilidad en el diseño del gráfico. En este sentido, es posible asignar categorías de atributos a los círculos concéntricos o dejarlos en blanco. Se puede dividir el diagrama y determinar de antemano el valor de cada sector, o bien prescindir de la segmentación. Otra ventaja es la posibilidad de presentar una visualización de la red sin nombres o categorías asociados a los alteri. De esta manera es posible utilizar las visualizaciones de red en presentaciones y publicaciones resguardando el anonimato de los participantes y demás personas involucradas.

Es posible utilizar estos programas como medio para diseñar las tarjetas de red, pero luego aplicarlas en papel (Olivier, 2013), o bien llenar el diagrama directamente en un computador (Noack \& Schmidt, 2013). Especialmente prácticos en este sentido son los computadores con touchscreen.

La aplicación digital es muy práctica, pero requiere un medio y personas afines a la tecnología. De este modo no siempre es posible utilizar este tipo de herramientas en contextos de investigación rurales o con personas mayores y de bajo nivel de educación. La aplicación también resulta complicada, si la red social es reconstruida por un grupo y no por una persona, ya que se plantea la pregunta acerca de quién ingresará los datos (Schönhuth, 2013).

Una aplicación de lápiz y papel alternativa a las tarjetas de red son los dibujos de red (Lutz, Behnken, \& Zinnecker, 2003; Scheibelhofer, 2006; Schönhuth, 2013). Los dibujos de red no son utilizados con mucha frecuencia, pues tienden a generar resistencia. El problema radica en que algunas personas adultas temen no dibujar lo suficientemente bien y por lo tanto tienden a reaccionar de manera negativa ante 
este tipo de aplicación (Lutz et al., 2003; Scheibelhofer, 2006; Schönhuth, 2013; Straus, 2002). No obstante, si el investigador logra superar la ansiedad inicial de los entrevistados, los dibujos de red pueden llegar a ser una valiosa herramienta para la reconstrucción de las redes sociales, ya que permiten mayor libertad de expresión subjetiva y por ende mayor espacio para la interpretación cualitativa de los datos.

Entre las limitaciones que supone el uso de dibujos de red se encuentra la baja comparabilidad de los dibujos entre sí, debido a la falta de elementos en común. Tampoco existe un procedimiento de análisis cualitativo específico para la interpretación de los dibujos. Hasta el momento, diversas investigaciones han resuelto este problema focalizando su atención en el texto transcrito de la entrevista y utilizando los dibujos como respaldo para el análisis (Scheibelhofer, 2006). Otros han analizado la forma en que fueron dibujados los objetos representados y el arreglo general de los distintos elementos del dibujo entre sí. Luego se ha contrastado lo dibujado con las narraciones que acompañaron el dibujo durante la entrevista (Lutz et al., 2003).

Otra alternativa para la reconstrucción de redes sociales corresponde a un tipo de herramientas hápticas e interactivas que ha sido utilizado en proyectos de ayuda al desarrollo local. Un ejemplo en este sentido es Net-Map (Schiffer \& Hauck, 2010). Net-Map ha sido usado para la reconstrucción de la red social de actores involucrados en una determinada problemática social, como lo puede ser el acceso a recursos locales. Los participantes utilizan discos de cartón y figuras para identificar a los actores involucrados. A continuación se reconstruye la capacidad de influencia y los intereses de cada actor y las relaciones de los alteri entre sí. El objetivo del instrumento ha sido en primer lugar el facilitar los procesos de aprendizaje de una comunidad y de apoyar a los participantes en la toma de decisiones. No obstante, también es posible utilizar esta herramienta con fines científicos.

Finalmente, queremos destacar otra herramienta que ha sido desarrollada especialmente para la reconstrucción de las redes sociales de niños (Picot \& Schroeder, 2007; Schroeder, Picot, \& Andresen, 2010). Se trata de un juego de redes socio-espaciales, en donde se reconstruyen los espacios cotidianos de los niños y las personas importantes que ellos conocen en estos lugares. Una de las razones que motivaron el desarrollo de este instrumento fue que las tarjetas de red resultaban demasiado abstractas para ser aplicadas en investigaciones con niños. El juego de redes socio-espaciales, en cambio, contiene varios elementos interactivos y lúdicos lo que lo hace accesible y atractivo para niños. 
A pesar de haber sido concebido como un instrumento para la reconstrucción de redes sociales de niños, el juego de redes socio-espaciales también permite su aplicación en adultos. En un proyecto sobre familias multilocales (Schier, 2009, 2013) hemos utilizado una adaptación del juego de redes socio-espaciales para su aplicación en adultos. Asimismo hemos desarrollado un método de análisis cualitativo de los datos obtenidos.

\section{Conclusiones}

Durante las últimas décadas ha aumentado la demanda por realizar estudios de redes sociales mediante el uso de una metodología cualitativa. Una aproximación cualitativa al estudio de redes sociales tiene la ventaja de poder dar cuenta de la perspectiva subjetiva y agencia de los actores involucrados. Sin embargo, el análisis cualitativo de las redes sociales constituye aún un ámbito incipiente y poco definido. De hecho, cuando se habla de un análisis cualitativo de redes por lo general se hace referencia a estudios de metodología mixta. En este marco, las técnicas cualitativas sirven como complemento para el análisis cuantitativo de los datos.

Dentro del contexto de la investigación cualitativa de las relaciones sociales ha adquirido especial importancia el uso de las tarjetas de red. Generalmente se combina la aplicación de este instrumento con una entrevista semi-estructurada o abierta. En el artículo hemos discutido la aplicación de un tipo particular de tarjeta de red denominado Ego.Net.QF. Gracias a la aplicación de esta herramienta es posible conocer de manera concreta las relaciones sociales en las cuales se insertan los entrevistados. La visualización de las redes sociales también constituye un importante estímulo reflexivo en el contexto de las entrevistas. Entre las limitaciones más relevantes de Ego.Net.QF destaca cierto desconcierto ante la solicitud de segmentar el diagrama y las restricciones que el tipo de visualización impone a una interpretación cualitativa de los datos.

Hemos visto que aún no existe un procedimiento de análisis cualitativo de redes sociales propiamente tal. Distintas aproximaciones cualitativas al análisis de redes han concentrado sus esfuerzos en la interpretación de los textos transcritos de las entrevistas y dejado de lado el análisis de las representaciones gráficas de las redes, situación que constituye actualmente una invitación a seguir explorando aproximaciones analíticas al respecto. 
La falta de claridad con respecto a cómo se realiza una investigación cualitativa de redes sociales en la práctica y la carencia de técnicas claramente definidas para la reconstrucción y análisis de este tipo de datos reflejan el estado inicial de desarrollo de la investigación cualitativa de las relaciones humanas. En este sentido podemos afirmar los estudios cualitativos de redes continúan siendo un importante laboratorio de experimentación, en el cual aún queda mucho por hacer.

\section{Referencias bibliográficas}

Aguilar, C. (2005). Visualización de redes personales en Sarajevo. REDES - Revista hispana para el análisis de redes sociales, 9(4), 1-14.

Cárdenas, A. (2012, August). Professional Women, Integration Work, and Risk Management in Contemporary Chile. International Sociological Association. Risk, Biography and Everyday Life, Buenos Aires.

Corsten, M. (2007). Lokales Sozialkapital und soziale Kognitionen über die eigene Stadt. Sozialwissenschaftliches Journal, 2(4), 60-83.

Diaz-Bone, R. (1997). Ego-zentrierte Netzwerkanalyse und familiale Beziehungssysteme. Wiesbaden: Deutscher Universitäts-Verlag.

Diaz-Bone, R. (2007). Gibt es eine qualitative Netzwerkanalyse? Forum Qualitative Sozialforschung, $8(1)$.

Flick, U. (2000). Triangulation in der qualitativen Forschung. In U. Flick, E. von Kardorff, \& S. Ines (Eds.), Qualitative Forschung. Ein Handbuch (pp. 309-318). Hamburg: Rowohlt.

Flick, U. (2004). Introducción a la investigación cualitativa. Madrid, Coruña: Ediciones Morata; Fundación Paideia Galiza.

Franke, K., \& Wald, A. (2006). Möglichkeiten der Triangulation quantitativer und qualitativer Methoden in der Netzwerkanalyse. In B. Hollstein \& F. Straus (Eds.), Qualitative Netzwerkanalyse. Konzepte, Methoden, Anwendungen. Wiesbaden: VS Verlag für Sozialwissenschaften.

Gualda Caballero, E. (2005). Pluralidad teórica, metodológica y técnica en el abordaje de las redes sociales: Hacia la "hibridación" disciplinaria. REDES - Revista hispana para el análisis de redes sociales, 9(1), 1-24.

Hein, K. (2006). Hybride Identitäten: Bastelbiografien im Spannungsverhältnis zwischen Lateinamerika und Europa. Bielefeld: transcript. 
Hein, K. (2012). Migración y Transición: Hijos de inmigrantes de origen latinoamericano en su transición de la escuela al trabajo en Chile. Si somos Americanos - Revista de estudios transfronterizos, 12(1), 101-126.

Hein, K. (2013). Vom Ausländer zum Jugendlichen: Interkulturelle Begegnungen, Identitätsarbeit und Handlungsfähgikeit bei jungen Migranten lateinamerikanischer Herkunft in Chile. Forum Gemeindepsychologie, 18(1).

Hollstein, B. (2006). Qualitative Methoden und Netzwerkanalyse: Ein Widerspruch? In B. Hollstein \& F. Straus (Eds.), Qualitative Netzwerkanalyse. Konzepte, Methoden, Anwendungen (pp. 11-36). Wiesbaden: VS Verlag für Sozialwissenschaften.

Hollstein, B. (2010). Qualitative Methoden und Mixed-Method-Designs. In C. Stegbauer \& R. Häußling (Eds.), Handbuch Netzwerkforschung (pp. 459-470). Wiesbaden: VS Verlag für Sozialwissenschaften.

Jansen, D. (2003). Einführung in die Netzwerkanalyse: Grundlagen, Methoden, Forschungsbeispiele. Opladen: Leske+Budrich.

Kahn, R. L., \& Antonucci, T. C. (1980). Convoys over the life course: Attachment, roles and social support. In P. B. Baltes \& O. G. Brim (Eds.), Life-span development and behaviour (pp. 383-405). New York: Academic Press.

Kesselring, S. (2006). Topographien mobiler Möglichkeitsräume: Zur soziomateriellen Netzwerkanalyse von Mobilitätspionieren. In B. Hollstein \& F. Straus (Eds.), Qualitative Netzwerkanalyse. Konzepte, Methoden, Anwendungen (pp. 333358). Wiesbaden: VS Verlag für Sozialwissenschaften.

Keupp, H., Abhe, T., Gmür, W., Höfer, R., Kraus, W., Mitzscherlich, B., \& Straus, F. (1999). Identitätskonstruktionen: Das Patchwork der Identität in der Spätmoderne. Reinbeck: Rowohlt.

Lamnek, S. (1995). Qualitative Sozialforschung. Weinheim: Beltz.

Lozares, C., \& Verd, J. M. (2008). La entrevista biográfico-narrativa como expresión contextualizada, situacional y dinámica de la red socio-personal. REDES - Revista hispana para el análisis de redes sociales, 15(6), 95-125.

Lutz, M., Behnken, I., \& Zinnecker, J. (2003). Narrative Landkarten: Ein Verfahren zur Rekonstruktion aktueller und biografisch erinnerter Lebensräume. In B. Friebertshauser \& A. Prengel (Eds.), Handbuch Qualitative Forschungsmethoden in der Erziehungswissenschaft (pp. 414-435). Weinheim: J uventa.

McCarty, C. (2002). Structure in Personal Networks. Journal of Social Structure, 3(1). 
McCarty, C., Molina, J. L., Aguilar, C., \& Rota, L. (2007). A Comparison of Social Network Mapping and Personal Network Visualization. Field Methods, 19(2), 145- 162 .

Mckether, W. L., Gluesing, J. C., \& Riopelle, K. (2009). From Interviews to Social Network Analysis: An approach for revealing social networks embedded in narrative data. Field Methods, 21(2), 154-180.

Noack, A., \& Schmidt, T. (2013). Netzwerk und Narration: Erfahrungen mit der computergestützten Erhebung qualitativer Egonetzwerke. In M. Schönhuth, M. Gamper, M. Kronenwett, \& M. Stark (Eds.), Visuelle Netzwerkforschung. Qualitative, quantitative und partizipative Zugänge (pp. 81-97). Bielefeld: transcript.

Olivier, C. (2013). Papier trotz Laptop?: Zur wechselseitigen Ergänzung von digitalen und haptischen Tools bei der qualitativen sozialen Netzwerkanalyse. In M. Schönhuth, M. Gamper, M. Kronenwett, \& M. Stark (Eds.), Visuelle Netzwerkforschung. Qualitative, quantitative und partizipative Zugänge (pp. 99119). Bielefeld: transcript.

Picot, S., \& Schroeder, D. (2007). Kinderpersönlichkeiten: Portraits von 12 Mädchen und Jungen. In World Vision Deutschland e.V. (Ed.), Kinder in Deutschland. 1. World Vision Kinderstudie (pp. 227-360). Frankfurt a.M.: Fischer.

Programa de las Naciones Unidas para el Desarrollo (PNUD). (2010). Informe Desarrollo Humano en Chile: Género: Los desafíos de la Igualdad. Santiago de Chile.

Programa de las Naciones Unidas para el Desarrollo (PNUD) \& Organización Internacional del Trabajo (OIT). (2009). Trabajo y Familia: Hacia nuevas formas de conciliación con corresponsabilidad social. Santiago de Chile.

Raab, J. (2010). Der "Harvard Breakthrough". In C. Stegbauer \& R. Häußling (Eds.), Handbuch Netzwerkforschung (pp. 29-37). Wiesbaden: VS Verlag für Sozialwissenschaften.

Rosenthal, G. (2005). Interpretative Sozialforschung. München: Juventa.

Scheibelhofer, E. (2006). Migration, Mobilität und Beziehung im Raum: Egozentrierte Netzwerkzeichnungen als Erhebungsmethode. In B. Hollstein \& F. Straus (Eds.), Qualitative Netzwerkanalyse. Konzepte, Methoden, Anwendungen (pp. 311332). Wiesbaden: VS Verlag für Sozialwissenschaften.

Schier, M. (2009). Familienleben auf Distanz. DJI Bulletin, (87), 22-23. 
Schier, M. (2013). Multilokale Wohnarrangements von Müttern, Vätern und ihren Kindern nach Trennung und Scheidung. In O. Schwedes (Ed.), Räumliche Mobilität in der Zweiten Moderne. Freiheit und Zwang bei Standortwahl und Verkehrsverhalten (pp. 189-212). Berlin: Lit Verlag.

Schiffer, E., \& Hauck, J. (2010). Net-Map: Collecting Social Network Data and Facilitating Network Learning through Participatory Influence Network Mapping. Field Methods, 22(3), 231-249.

Schönhuth, M. (2013). Landkarten sozialer Beziehungen: Partizipativ-visuelle Datenerhebung mit haptischen und elektronischen Werkzeugen: Entwicklungen Typen - Möglichkeiten - Grenzen. In M. Schönhuth, M. Gamper, M. Kronenwett, \& M. Stark (Eds.), Visuelle Netzwerkforschung. Qualitative, quantitative und partizipative Zugänge (pp. 59-78). Bielefeld: transcript.

Schönhuth, M., \& Gamper, M. (2013). Visuelle Netzwerkforschung: Eine thematische Annäherung. In M. Schönhuth, M. Gamper, M. Kronenwett, \& M. Stark (Eds.), Visuelle Netzwerkforschung. Qualitative, quantitative und partizipative Zugänge (pp. 9-32). Bielefeld: transcript.

Schroeder, D., Picot, S., \& Andresen, S. (2010). Die qualitative Studie: 12 Portäts von Kinderpersönlichkeiten. In World Vision Deutschland e.V. (Ed.), Kinder in Deutschland 2010. 2. World Vision Kinderstudie (pp. 223-234). Frankfurt a.M.: Fischer.

Schütz, I. (2006). Quantitative und qualitative Veränderungen in den sozialen Netzwerken junger Migranten: Ergebnisse einer Langzeitstudie. In B. Hollstein \& F. Straus (Eds.), Qualitative Netzwerkanalyse. Konzepte, Methoden, Anwendungen. Wiesbaden: VS Verlag für Sozialwissenschaften.

Straus, F. (1994). Netzwerkanalyse: Egozentrierte Netzwerkkarten als Instrument zur Erhebung von sozialen Beziehungen in qualitativen Interviews. München.

Straus, F. (2002). Netzwerkanalysen: Gemeindepsychologische Perspektive für Forschung und Praxis. Wiesbaden: Deutscher Universitäts-Verlag.

Straus, F. (2006). Entwicklungslabor qualitative Netzwerkforschung. In B. Hollstein \& F. Straus (Eds.), Qualitative Netzwerkanalyse. Konzepte, Methoden, Anwendungen (pp. 481-494). Wiesbaden: VS Verlag für Sozialwissenschaften.

Straus, F. (2010). Netzwerkkarten: Netzwerke sichtbar machen. In C. Stegbauer \& R. Häußling (Eds.), Handbuch Netzwerkforschung (pp. 527-538). Wiesbaden: VS Verlag für Sozialwissenschaften. 
Straus, F. (2013). "Das Unsichtbare sichtbar machen": 30 Jahre Erfahrungen mit qualitativen Netzwerkanalysen. In M. Schönhuth, M. Gamper, M. Kronenwett, \& M. Stark (Eds.), Visuelle Netzwerkforschung. Qualitative, quantitative und partizipative Zugänge (pp. 33-58). Bielefeld: transcript.

Todaro, R., \& Yañez, S. (2004). El trabajo se transforma: Relaciones de producción y relaciones de género. Santiago de Chile: Centro de Estudios de la Mujer (CEM).

Wolf, C. (2010). Egozentrierte Netzwerke: Datenerhebung und Datenanalyse. In C. Stegbauer \& R. Häußling (Eds.), Handbuch Netzwerkforschung. Wiesbaden: VS Verlag für Sozialwissenschaften. 